\title{
Biomarkers of mucinous tumors of the ovary
}

\author{
Goli Samimi $^{\mathrm{a}, \mathrm{b}}$, Laurent Ozbun ${ }^{\mathrm{b}}$, Michael E. Johnson ${ }^{\mathrm{c}}$, Samuel C. Mok ${ }^{\mathrm{c}}$ and Michael J. Birrer ${ }^{\mathrm{b}}$ \\ ${ }^{a}$ Cancer Prevention Fellowship Program, National Cancer Institute, Bethesda, MD 20892, USA \\ ${ }^{\mathrm{b}}$ Cell and Cancer Biology Branch, Center for Cancer Research, National Cancer Institute, Bethesda, MD 20892, \\ USA \\ ${ }^{\mathrm{c}}$ Laboratory of Gynecologic Oncology, Brigham and Women's Hospital Harvard Medical School, Boston, MA \\ 02115, USA
}

\section{Introduction}

Ovarian tumors are thought to arise from the surface epithelium of the ovary and are classified into four major histologic classes: serous, mucinous, endometrioid, clear cell and (reviewed in [1]). The molecular and cellular mechanisms of ovarian tumorigenesis remain unknown and are likely to vary between the different histotypes of ovarian cancer [2]. 12-15\% of all ovarian neoplasms are mucinous ovarian tumors. The majority (75\%) of mucinous tumors are benign, $15 \%$ are malignant and the remaining $10 \%$ of tumors are borderline or low malignant potential (LMP).

Mucinous ovarian tumors possess clinical characteristics that distinguish them from ovarian tumors of other histotypes. A proportion of mucinous ovarian tumors are believed to undergo a unique process of sequential development (Fig. 1); beginning as a benign cyst and progressing to an LMP tumor before developing into an adenocarcinoma [3,4]. This model of tumor progression is specific to the mucinous histotype of ovarian tumors (both low- and high-grade tumors) and differentiates them from ovarian tumors of other histological origins. It is supported in part by the observation of the co-existence of benign, LMP and malignant pathologies within the same tumor by electron microscopy and histological studies $[3,5,6]$. The presence of identical $K$-ras mutations in these different pathologic regions of the tumor lends further support to the tumor progression model [7]. Hierarchical clustering and binary tree analysis of gene expression profiles of mucinous tumors of the ovary revealed that muci- nous cystadenomas were distinct from the other mucinous samples while mucinous LMP and low-grade mucinous adenocarcinomas were nearly indistinguishable from each other [8]. When serous tumors were included in the analysis, the results demonstrated that while mucinous LMP and low-grade mucinous adenomcarcinomas remained nearly indistinguishable, serous LMP and serous adenocarcinoma tumors were grouped in distinct branches [8]. These results add further support to the conceived model for mucinous tumor progression. This model suggests that mucinous tumors of the ovary differ from other histotypes in that they do not develop de novo but rather develop from benign or LMP tumors, thus indicating the potential presence of biomarkers that may be unique to the mucinous histotype.

Patients with LMP mucinous tumors in general have a good prognosis after surgery since most of these tumors are diagnosed at an early stage and the 5-year cumulative survival rate for women with LMP is $97.0 \%$ $( \pm 1.5 \%)$ [9]. However, women with advance stage mucinous adenocarcinoma tumors respond poorly to standard platinum-based chemotherapy compared to women with ovarian tumors of other histotypes and have a significantly reduced 5-year survival rate of $67.4 \%$ [9-13].

The diagnosis of mucinous carcinoma of the ovary is challenging. Many mucinous tumors have a mixed histology (such as a high frequency of intestinal differentiation) and are difficult to recognize with standard pathology (reviewed in [14]). It is believed that 7 to $17 \%$ of all ovarian tumors are metastatic rather than pri- 




Fig. 1. Mucinous tumor of the ovary containing increasing degrees aggressiveness (presented with permission of Samuel Mok).

mary [15] and the histologic features of metastatic mucinous ovarian carcinomas can be very similar to those of primary mucinous ovarian tumors [16]. Histopathology in combination with gross examination can be useful in distinguishing primary and metastatic mucinous tumors. Features including bilaterality, microscopic surface involvement of epithelial cells, nodular pattern of growth, an infiltrative invasive pattern and growth in the ovarian hilus are favored in metastatic cases while size $>10 \mathrm{~cm}$, a smooth tumor surface, benign or borderline areas, an expansile invasive pattern, microscopic cysts $>2 \mathrm{~mm}$, complex papillary epithelial growth and intraluminal necrotic material are favored in primary tumors [17]. However, these guidelines are not completely reliable and may not be applicable to late-stage mucinous tumors of the ovary.

The clinical characteristics of mucinous ovarian tumors (better survival rates for patients with LMP mucinous ovarian tumors versus those with advanced mucinous adenocarcinoma, differential response to chemotherapy and similar histologies between primary and metastatic mucinous tumors of the ovary) necessitates the identification and validation of biomarkers that can improve specific detection, diagnosis and prognosis of mucinous tumors of the ovary. DNA microarrays permit simultaneous, comprehensive, genomewide monitoring of gene expression. Gene expression profiling has been applied in cancer research to identify important genes or pathways that molecularly classify tumors, contribute to carcinogenesis, predict chemoresponse or serve as biomarkers of clinical characteristics including diagnosis and prognosis (reviewed in [18]). These studies have provided a basis for the development of personalized medicine (reviewed in [19]).

\section{Gene expression profiling for identification of mucinous-specific biomarkers}

Gene expression profiling has been utilized to identify potential tumor biomarkers that can be used in clinical applications (including detection, diagnosis and prognosis) in a variety of tumor types including prostate [20-23], colon [24], breast [25,26] and ovarian cancer [27-31]. More specifically, gene expression profiling has been applied to identify genes that differentiate between different histotypes of ovarian cancer as well as genes expressed in specific subtypes that may play a role in their particular clinicopathology.

In a small study, Ono et al. compared gene expression patterns in ovarian tumor samples versus their corresponding non-cancerous ovarian tissue as well as genes that were differentially expressed between serous and mucinous tumors [32]. The authors analyzed gene expression profiles of five serous and four mucinous ovarian adenocarcinomas and patient-matched normal ovarian tissue using a custom-made cDNA microarrays consisting of 9,121 genes. The study revealed 115 differentially expressed genes between mucinous and serous tumors [32]. Creatinine kinase B, myosin heavy polypeptide 11 and A28-RGS14p, a p53 target gene and regulator of $\mathrm{G}$ protein signaling, were expressed at higher levels in mucinous tumors [32]. Overexpression of A28-RGS14p has previously been shown to inhibit $G$ protein-coupled receptor activation of the mitogen-activated protein kinase signaling pathway in various carcinoma cell lines [33]. Thus, A28-RGS14 may play a role in the progression of mucinous tumors, presumably through regulation of cellular signaling in response to growth factors acting through $G$ proteincoupled receptor pathways. 
In a larger study, Marquez et al. investigated the molecular alterations contributing to differences between the histotypes of ovarian cancer [34]. The authors utilized the Affymetrix Human Genome U95 platform to compare gene expression profiles of 50 total epithelial ovarian cancer samples, including $9 \mathrm{mu}-$ cinous tumors, with profiles of normal ovarian surface epithelium taken directly from patients. Hierarchical clustering of all samples clearly distinguished most of the mucinous samples from the rest of the tumors and, interestingly, they were clustered in a branch with normal samples [34]. Further analysis of genes whose expression was differentially expressed by at least 2-fold between the histotypes and differed from normal ovarian surface epithelium revealed 46 genes differentially expressed in mucinous tumors. Based on quantitative real-time PCR validation, the most specific biomarkers for mucinous tumors versus ovarian tumors of other histotypes were TFF1 (involved in immune response, cellular defense, mucosal maintenance and repair), AGR2, LGALS4 and CEACAM6 (all involved in extracellular matrix formation and cell adhesion) and CTSE (involved in metabolism and proteolysis). The AGR2 gene is an estrogen-responsive gene whose role in mucinous ovarian tumorigenesis is currently unclear but has been shown to be over-expressed in prostate cancer [35] and is involved in breast cancer metastasis [36]. Schwartz et al. reported that TFF1 gene and protein expression is specifically up-regulated in mucinous tumors [37]. TFF1 is a secreted peptide that belongs to the trefoil factor family and its gene expression is induced by estrogen and FGF2. It regulates several signal transduction pathways including the PI3kinase/Akt pathway, the MAPK pathway, and other pathways involved in cell progression and transformation (reviewed by [38]). With the exception of CTSE, these genes have been identified as mucinous-specific ovarian tumor biomarkers by other studies discussed in this review.

Heinzelmann-Schwarz et al. studied the genetic basis of mucinous ovarian carcinomas and identified potential markers that distinguish mucinous tumors from ovarian tumors of other histotypes [39]. The authors utilized a customized Affymetrix oligonucleotide microarray to profile gene expression from 3 mucinous ovarian tumors, 4 mucinous borderline tumors, 8 endometrioid ovarian tumors, 31 serous ovarian tumors and 4 normal ovary samples [39]. Forty genes were identified as up-regulated and 4 genes were identified as down-regulated in mucinous ovarian tumors compared to normal ovarian tissues. Of the 40 genes up-regulated in mucinous ovarian tumors, 13 genes were unique to mucinous and not found to be differentially expressed in ovarian tumors of other histotypes. These potential mucinous-specific biomarkers include LGALS4, MUC13, MUCDHL, CEACAM5, CALML4, ARHGAP27, NMES1, BCLP, CDN17 and TFF1 [39]. The authors demonstrate that mucinous ovarian tumors over-express genes associated with mucin production and intestinal function. This study identified genes that were also found in other studies such as TFF1, which has been shown in previous studies to be up-regulated in other mucinous adenocarcinomas, including the study by Marquez et al. discussed above [34]. Other genes identified as specifically up-regulated in mucinous tumors both in this study as well as in the study by Marquez et al. include CEACAM6, a member of the carcinoembryonic antigen family shown to be over-expressed in many different cancers including ovarian, breast and colon cancer [40], and LGALS4, an intestinal-type cell adhesion molecule shown to be over-expressed in several other epithelial cancers including intestinal, breast and liver $[41,42]$. LGALS4 is a potential candidate for an early mucinous biomarker, due to its lack of expression in normal ovary and its high expression in mucinous cysts and LMP tumors. LGALS4 is not a secreted protein and therefore its clinical relevance as a diagnostic biomarker may be limited; however the protein contains an extracellular component that may be useful for detection and measurement $[39,42]$. CEACAM6 is a membrane-associated protein that interacts with other members of the CEA family and integrin receptors to promote cellular adhesion [43]. However, because it has been shown to be over-expressed in multiple tumors, its use as a mucinous-specific diagnostic biomarker may be limited and has not been extensively investigated.

Wamunyokoli et al. have conducted the largest profiling study to date on mucinous tumors of the ovary. They analyzed multiple mucinous specimens of differing histologic grade, all of which have been microdissected. The study sought to uncover potential markers that distinguish between the various mucinous histological subtypes [8]. Gene expression profiles of 25 mucinous tumors were compared to gene expression profiles of 40 serous tumors and 10 normal ovarian surface epithelial samples using the Affymetrix Human Genome U133 Plus 2.0 platform. Unsupervised hierarchical clustering of all samples revealed 2 arms; normal ovarian surface epithelial samples and serous LMP tumors clustered in different branches on a single arm 
while serous adenocarcinomas and mucinous tumors clustered in different branches on the second arm, with high-grade mucinous adenocarcinomas forming a distinct branch. These results are suggestive of a more aggressive phenotype associated with mucinous tumors and suggest the existence of a subset of genes that are co-regulated in high-grade serous adenocarcinomas and mucinous tumors. A list of genes whose expression is unique to the mucinous phenotype was extracted by comparing genes that are differentially expressed in mucinous or serous tumors versus normal ovarian surface epithelium and selecting only those genes that are up-regulated in mucinous tumors. From this list of genes, potential biomarkers with clinical relevance were identified based on whether they were secreted or membrane-bound. The potential biomarkers identified for mucinous tumors were AGR2, FAM3B, MUC13, IER3, ADD3 and SLC12A2.

Further analyses were performed to identify genes that may contribute to mucinous ovarian tumor progression and reveal potential markers that distinguish between the various mucinous histological subtypes. NET1, ERBB3, H63, TSPAN8, ANXA2, GLTSCR2 and CCAR1 were identified as up-regulated in both LMP and adenocarcinoma mucinous tumors but not in cystadenoma; TPT1, CTNNA and TACSTD1 were upregulated in LMP tumors only and TMEM50A, RAC1 and CTTN were up-regulated in adenocarcinoma tumors only. Some of these genes have been demonstrated in previous studies to be related to tumor progression. Over-expression of NET1in NIH3T3 cells results in increased tumorigenicity [44] and ERBB3 promotes growths and invasiveness in lung adenocarcinoma [45]. RAC1 is a rhoGTPase that has been shown to be involved in ovarian tumor migration and progression [46]. Therefore, these genes may play a role in transformation of mucinous tumors. This study was successful in identifying several potential novel markers that may be useful in distinguishing mucinous ovarian tumors from ovarian tumors of other histotypes as well as distinguishing between the various mucinous subtypes.

\section{Protein expression analysis for identification of mucinous-specific biomarkers}

Gene expression profiling can be used successfully to identify potential tumor biomarkers. However, it is clear that the most clinically relevant biomarkers are those that are secreted during development and in early stage of tumor growth. Recent studies have applied such techniques as immunohistochemistry and proteomic techniques to identify putative ovarian cancer biomarkers (reviewed in [15]). While the number of these studies is limited, they reveal meaningful information regarding epithelial ovarian carcinogenesis progression, diagnosis and prognosis.

In an effort to classify different histotypes of ovarian epithelial tumors and identify surrogate biomarkers indicative of ovarian tumors, An et al. utilized 2dimensional PAGE proteomics to create distance trees based on variations of protein expression patterns within and between 12 ovarian epithelial tumors including 4 serous, 5 mucinous and 3 endometrioid tumors [47]. The results of the distance map trees demonstrated that while the tumor samples were separate from normal ovary, mucinous carcinomas exhibited protein expression patterns most closely related to normal ovarian samples. The most aggressive mucinous tumor types were separate from the LMP tumors, yet more similar to each other than to other ovarian epithelial tumor histotypes [47]. Six potential biomarkers (NM23-H1, annexin 1 , protein phosphatase-1, ferritin light chain, proteasome $\alpha-6$ and NAGK) were identified whose expressions were increased by at least 2-fold in ovarian tumor samples compared to normal ovary tissues. These protein products were not specific for the mucinous histotype and thus may only be useful as general biomarkers for the diagnosis of ovarian cancer. Nonetheless, the findings of this study suggest that proteomic profiling can be a valuable tool for the future identification of potential biomarkers that are unique to the mucinous histotype.

\section{Identification of biomarkers to distinguish between primary and metastatic mucinous tumors}

Primary ovarian mucinous tumors are difficult to diagnose as they are often confused with metastatic mucinous cancer resulting from other origins. As a result of their mixed histology, clinicians and pathologists must also rely on other clinical features including bilaterality, tumor dissemination pattern as well as cytology/histology to reliably ascertain the origin of the tumor $[14,17,48,49]$. As a result, several immunohistochemcial studies have been performed to identify biomarkers that can aid in distinguishing between primary and metastatic mucinous tumors. 
A study published by $\mathrm{Ji}$ et al. sought to identify biomarkers that can differentiate between primary and metastatic mucinous ovarian carcinomas. The authors examined the immunohistochemical expression of cytokeratins CK7 and CK20, the nuclear transcription factor DPC4 (also known as SMAD4) and the gastric mucin MUC5AC in 57 primary ovarian mucinous tumors (41 LMP and 16 malignant) and 46 metastatic mucinous tumors of the ovary [50]. The findings demonstrated that almost all (98-100\%) primary ovarian mucinous tumors expressed CK7, DPC4 and MUC5AC while a smaller portion (68\%) expressed CK20. Some of these markers were also expressed in a majority of metastatic tumors of the ovary (i.e. $100 \%$ of pancreatic cancer cases expressed CK7 and 100\% of colorectal cancers expressed CK20), suggesting that these markers alone may not be useful in distinguishing between primary ovarian mucinous carcinoma and metastatic mucinous carcinomas. Some of the examined biomarkers were expressed to a lesser extent in metastatic tumors of the ovary. Only $33 \%$ of colorectal carcinomas expressed CK7 and/or MUC5AC and only 54\% of pancreatic carcinomas expressed DPC4. The results of the study suggest that combined expression of both CK7 and CK20 is the most appropriate to distinguish between primary mucinous tumors of the ovary and metastatic colorectal tumors while loss of DPC4 expression can distinguish between primary mucinous tumors of the ovary and metastatic pancreatic carcinomas [50].

It has been reported that approximately $40 \%$ of metastatic tumors of the ovary originates from colorectal cancer [16]. Because the development of colon cancer relies on Wnt signaling through $\beta$-catenin [51], Chou et al. sought to determine whether $\beta$-catenin expression could also be used to differentiate between ovarian mucinous carcinoma and metastatic colorectal adenocarcinoma [52]. The study analyzed expression of $\beta$-catenin, along with CK7, CK20 and CEA in 43 primary ovarian mucinous carcinomas and 23 metastatic colorectal adenocarcinomas by immunohistochemistry [52]. Expression of $\beta$-catenin was detected in most $(83 \%)$ of metastatic colorectal adenocarcinomas and only $9 \%$ of primary ovarian mucinous carcinomas. Consistent with the Ji study [50], CK7 was expressed in a majority $(79 \%)$ of mucinous ovarian tumors and only $23 \%$ of colorectal samples while CK20 was expressed in most colorectal adenocarcinomas (87\%) and only $44 \%$ of mucinous ovarian tumors expressed the protein. In addition, CEA was found to be expressed in $70 \%$ of mucinous carcinomas, similar to gene expres- sion profile studies [34,39]. However, CEA was also expressed in $95 \%$ of colorectal carcinomas, suggesting that while it may be able to differentiate mucinous tumors of the ovary from other ovarian histotypes, it does not serve as an appropriate biomarker to distinguish between primary and metastatic mucinous tumors. The results of this study support the conclusion that CK7, CK20 and $\beta$-catenin expression may be used to distinguish between primary mucinous tumors of the ovary and metastatic colorectal tumors [52].

Another gene that has been studied as a potential biomarker for metastatic mucinous tumors is CDX2, a transcription factor expressed in most colorectal carcinomas that is involved in intestinal development, proliferation and differentiation [53,54]. Several studies have examined its expression in primary mucinous tumors of the ovary, with inconsistent results (reviewed in [14]). Some have suggested that expression of CDX2 in mucinous tumors of the ovary is dependent on cell type (endocervical or intestinal) [55,56]. A few studies have been performed that have analyzed expression of CDX2 in coordination with other, better-established mucinous ovarian tumor biomarkers including CK7, CK20 and $\beta$-catenin in an effort to increase their sensitivity. Most studies detect CDX2 expression in all colorectal carcinomas examined; however expression of CDX2 in mucinous tumors of the ovary varies in the different studies. One study examined the expression of CDX2, CK7 and CK20 by immunohistochemistry and CDX2 was detected in $100 \%$ of mucinous tumors of the ovary [57]. Other immunohistochemical studies detected CDX2 in only $40 \%$ [58] and $21 \%$ of mucinous tumors of the ovary [56]. It appears that CDX2 alone is not a useful biomarker for distinguishing between primary and metastatic mucinous tumors of the ovary.

\section{Challenges of applications of biomarkers to clinical issues of mucinous tumors of the ovary}

Gene expression profiling and proteomic approaches have been demonstrated to be useful techniques for the identification of mucinous-specific biomarkers. Several biomarkers identified by gene expression profiling (including TFF1, AGR2, LGALS4, CEACAM5, CEACAM6 and MUC13) are specifically present in mucinous tumors of the ovary and could be useful in distinguishing mucinous ovarian cancers from ovarian tumors of other histotypes. With the exception of AGR2, these markers are also expressed to different degrees in tumors of other origins, particularly those from the gas- 
Table 1

Summary of biomarkers discussed in this review

\begin{tabular}{|c|c|c|c|c|}
\hline Biomarker & Gene/Protein & $\begin{array}{l}\text { Ovarian cancer } \\
\text { histotypes }\end{array}$ & $\begin{array}{l}\text { Other mucinous } \\
\text { carcinomas }\end{array}$ & Reference \\
\hline $\begin{array}{l}\text { TFF1 } \\
\text { (Trefoil Factor Family 1) }\end{array}$ & Gene, Protein & Up-regulated in mucinous & $\begin{array}{l}\text { Expressed in colorectal } \\
\text { carcinomas [38] }\end{array}$ & {$[34,37,39]$} \\
\hline $\begin{array}{l}\text { AGR2 } \\
\text { (Anterior Gradient } \\
2 \text { homolog) }\end{array}$ & Gene & Up-regulated in mucinous & $\begin{array}{l}\text { Down-regulated in } \\
\text { colorectal carcinomas } \\
\text { versus normal [59] }\end{array}$ & {$[8,34]$} \\
\hline $\begin{array}{l}\text { LGALS4 } \\
\text { (Galectin 4) }\end{array}$ & Gene, Protein & Up-regulated in mucinous & $\begin{array}{l}\text { Expressed in colorectal } \\
\text { carcinomas [41] }\end{array}$ & {$[34,39]$} \\
\hline $\begin{array}{l}\text { CEACAM5 } \\
\text { CEACAM6 } \\
\text { (Carcinoembryonic } \\
\text { antigen-related cell } \\
\text { adhesion molecule) }\end{array}$ & Gene & Up-regulated in mucinous & $\begin{array}{l}\text { Expressed in } 95 \% \text { colorectal } \\
\text { carcinomas [52] }\end{array}$ & {$[34,39]$} \\
\hline $\begin{array}{l}\text { MUC13 } \\
\text { (Mucin 13) }\end{array}$ & Gene & Up-regulated in mucinous & $\begin{array}{l}\text { Expressed in colorectal } \\
\text { carcinomas }[60]\end{array}$ & {$[8,39]$} \\
\hline $\begin{array}{l}\text { CK7 } \\
\text { (Cytokeratin 7) }\end{array}$ & Protein & $\begin{array}{l}\text { Expressed in most mucinous (79- } \\
100 \%)\end{array}$ & $\begin{array}{l}\text { Expressed in some colorectal } \\
\text { carcinomas }(23-33 \%)\end{array}$ & {$[50,52]$} \\
\hline $\begin{array}{l}\text { CK20 } \\
\text { (Cytokeratin 20) }\end{array}$ & Protein & $\begin{array}{l}\text { Expressed in some mucinous (44- } \\
68 \%)\end{array}$ & $\begin{array}{l}\text { Expressed in most colorectal } \\
\text { carcinomas }(87-100 \%)\end{array}$ & {$[50,52]$} \\
\hline $\mathrm{CDX} 2$ & Protein & Inconsistent expression in mucinous & Expressed in all colorectal carcinomas & {$[56-58]$} \\
\hline $\begin{array}{l}\text { DPC4 } \\
\text { (Deleted in } \\
\text { pancreatic cancer } \\
\text { locus 4; SMAD4) }\end{array}$ & Protein & Expressed in all mucinous (100\%) & $\begin{array}{l}\text { Expressed in } 89 \% \text { colorectal and } 54 \% \\
\text { pancreatic carcinomas }\end{array}$ & {$[50]$} \\
\hline$\beta$-catenin & Protein & Expressed in only $9 \%$ mucinous & $\begin{array}{l}\text { Expressed in } 83 \% \text { colorectal } \\
\text { carcinomas }\end{array}$ & {$[52]$} \\
\hline
\end{tabular}

trointestinal track (summarized in Table 1). Therefore, their use as biomarkers to distinguish between primary mucinous tumors of the ovary and metastatic mucinous tumors is limited.

Taking into account the immunohistochemical findings presented in this review, it appears that the most valuable approach to biomarker screening for differentiating primary mucinous tumors of the ovary and metastatic mucinous tumors is to compare coordinated expression of multiple biomarkers. Currently, the most commonly used biomarkers are CK7 and CK20, with a CK7+/CK20- profile being indicative of primary mucinous tumors of the ovary while a CK7-/CK20+ profile is indicative of a metastatic mucinous tumor (reviewed in [49]). These profiles are limited as CK7 has been shown to be expressed in some colorectal carcinomas and CK20 has been shown to be expressed in some mucinous ovarian tumors (summarized in Table 1). These profiles could be made more reliable by applying additional biomarkers. As an example, AGR2 gene expression has been shown to be up-regulated in mucinous tumors and down-regulated in colorectal cancers. Therefore, coordinated expression of CK7, CK20 and AGR2 could potentially be more reliable to distinguish between mucinous tumors of the ovary and metastatic tumors from the colon. A CK7+/CK20-/AGR2+ profile would be more indicative of primary mucinous tu- mors of the ovary while a CK7-/CK20+/AGR2- profile would be more indicative of a metastatic mucinous colorectal tumor. Expression of multiple biomarkers coordinated with the gross/histologic profiles as described above (e.g. bilaterality, invasive patterns and size) could further refine the distinction between mucinous tumors of the ovary and metastatic mucinous tumors.

\section{Conclusions}

Similarities between primary and metastatic mucinous tumors of the ovary make them difficult to accurately diagnosis. The identification of biomarkers that can distinguish primary from metastatic mucinous tumors and/or are unique to mucinous tumors of the ovary could be used in combination with histologic features of the tumor to significantly improve early diagnosis, and consequently improve patient prognosis.

Gene expression profiling and proteomic analysis are valuable tools for molecular characterization of various mucinous tumors of the ovary and the identification of mucinous-specific biomarkers (summarized in Table 1). All potential markers will need extensive validation before they can be used clinically to improve detection and diagnosis. 


\section{References}

[1] M. Scott and W.G. McCluggage, Current concepts in ovarian epithelial tumorigenesis: correlation between morphological and molecular data, Histol Histopathol 21(1) (2006), 81-92.

[2] M. Pieretti et al., Genetic alterations distinguish different types of ovarian tumors, Int J Cancer 64(6) (1995), 434-440.

[3] R. Scully, D. Bell and G. Abu-Jardeh, Update on Early Ovarian Cancer and Cancer Developing in Benign Ovarian Tumours, 1995, Chapman and Hall Medical: London.

[4] I.M. Shih and R.J. Kurman, Ovarian tumorigenesis: a proposed model based on morphological and molecular genetic analysis, Am J Pathol 164(5) (2004), 1511-1518.

[5] L.E. Puls et al., Transition from benign to malignant epithelium in mucinous and serous ovarian cystadenocarcinoma, $G y$ necol Oncol 47(1) (1992), 53-57.

[6] F. Stenback, Benign, borderline and malignant serous cystadenomas of the ovary. A transmission and scanning electron microscopical study, Pathol Res Pract 172(1-2) (1981), $58-72$.

[7] A.P. Garrett et al., k-ras mutation may be an early event in mucinous ovarian tumorigenesis, Int J Gynecol Pathol 20(3) (2001), 244-251.

[8] F.W. Wamunyokoli et al., Expression profiling of mucinous tumors of the ovary identifies genes of clinicopathologic importance, Clin Cancer Res 12(3 Pt 1) (2006), 690-700.

[9] M.E. Sherman et al., Survival among women with borderline ovarian tumors and ovarian carcinoma: a population-based analysis, Cancer 100(5) (2004), 1045-1052.

[10] V. Hess et al., Mucinous epithelial ovarian cancer: a separate entity requiring specific treatment, J Clin Oncol 22(6) (2004), 1040-1044.

[11] C.L. Kosary, FIGO stage, histology, histologic grade, age and race as prognostic factors in determining survival for cancers of the female gynecological system: an analysis of 1973-87 SEER cases of cancers of the endometrium, cervix, ovary, vulva, and vagina, Semin Surg Oncol 10(1) (1994), 31-46.

[12] A.P. Makar et al., The prognostic significance of residual disease, FIGO substage, tumor histology, and grade in patients with FIGO stage III ovarian cancer, Gynecol Oncol 56(2) (1995), 175-180.

[13] G.A. Omura et al., Long-term follow-up and prognostic factor analysis in advanced ovarian carcinoma: the Gynecologic Oncology Group experience, J Clin Oncol 9(7) (1991), 11381150 .

[14] W.R. Hart, Mucinous tumors of the ovary: a review, Int $J$ Gynecol Pathol 24(1) (2005), 4-25.

[15] P.M. Baker and E. Oliva, Immunohistochemistry as a tool in the differential diagnosis of ovarian tumors: an update, Int $J$ Gynecol Pathol 24(1) (2005), 39-55.

[16] D. Daya, L. Nazerali and G.L. Frank, Metastatic ovarian carcinoma of large intestinal origin simulating primary ovarian carcinoma. A clinicopathologic study of 25 cases, Am J Clin Pathol 97(6) (1992), 751-758.

[17] K.R. Lee and R.H. Young, The distinction between primary and metastatic mucinous carcinomas of the ovary: gross and histologic findings in 50 cases, Am J Surg Pathol 27(3) (2003), 281-292.

[18] S. Mohr et al., Microarrays as cancer keys: an array of possibilities, J Clin Oncol 20(14) (2002), 3165-3175.

[19] M.E. Burczynski et al., Clinical pharmacogenomics and transcriptional profiling in early phase oncology clinical trials, Curr Mol Med 5(1) (2005), 83-102.
[20] J.H. Bull et al., Identification of potential diagnostic markers of prostate cancer and prostatic intraepithelial neoplasia using cDNA microarray, Br J Cancer 84(11) (2001), 1512-1519.

[21] J.C. Miller et al., Antibody microarray profiling of human prostate cancer sera: antibody screening and identification of potential biomarkers, Proteomics 3(1) (2003), 56-63.

[22] M.A. Rubin et al., Tissue microarray sampling strategy for prostate cancer biomarker analysis, Am J Surg Pathol 26(3) (2002), 312-319.

[23] T. Zellweger et al., Tissue microarray analysis reveals prognostic significance of syndecan-1 expression in prostate cancer, Prostate 55(1) (2003), 20-29.

[24] M.B. Resnick et al., Epidermal growth factor receptor, c-MET, beta-catenin, and p53 expression as prognostic indicators in stage II colon cancer: a tissue microarray study, Clin Cancer Res 10(9) (2004), 3069-3075.

[25] S.K. Gruvberger-Saal et al., Predicting continuous values of prognostic markers in breast cancer from microarray gene expression profiles, Mol Cancer Ther 3(2) (2004), 161-168.

[26] M. Lacroix et al., A low-density DNA microarray for analysis of markers in breast cancer, Int J Biol Markers 17(1) (2002), $5-23$.

[27] H.G. Huddleston et al., Clinical applications of microarray technology: creatine kinase B is an up-regulated gene in epithelial ovarian cancer and shows promise as a serum marker, Gynecol Oncol 96(1) (2005), 77-83.

[28] B.C. Lee et al., Microarray analysis of differentially expressed genes associated with human ovarian cancer, Int J Oncol 24(4) (2004), 847-851.

[29] S.C. Mok et al., Prostasin, a potential serum marker for ovarian cancer: identification through microarray technology, J Natl Cancer Inst 93(19) (2001), 1458-1464.

[30] V. Ouellet et al., Tissue array analysis of expression microarray candidates identifies markers associated with tumor grade and outcome in serous epithelial ovarian cancer, Int J Cancer 119(3) (2006), 599-607.

[31] V. Shridhar et al., Genetic analysis of early- versus late-stage ovarian tumors, Cancer Res 61(15) (2001), 5895-5904.

[32] K. Ono et al., Identification by cDNA microarray of genes involved in ovarian carcinogenesis, Cancer Res 60(18) (2000), 5007-5011.

[33] L. Buckbinder et al., The p53 tumor suppressor targets a novel regulator of G protein signaling, Proc Natl Acad Sci USA 94(15) (1997), 7868-7872.

[34] R.T. Marquez et al., Patterns of gene expression in different histotypes of epithelial ovarian cancer correlate with those in normal fallopian tube, endometrium, and colon, Clin Cancer Res 11(17) (2005), 6116-6126.

[35] J.S. Zhang et al., AGR2, an androgen-inducible secretory protein overexpressed in prostate cancer, Genes Chromosomes Cancer 43(3) (2005), 249-259.

[36] D. Liu et al., Human homologue of cement gland protein, a novel metastasis inducer associated with breast carcinomas, Cancer Res 65(9) (2005), 3796-3805.

[37] D.R. Schwartz et al., Gene expression in ovarian cancer reflects both morphology and biological behavior, distinguishing clear cell from other poor-prognosis ovarian carcinomas, Cancer Res 62(16) (2002), 4722-4729.

[38] S. Emami et al., Trefoil factor family (TFF) peptides and cancer progression, Peptides 25(5) (2004), 885-898.

[39] V.A. Heinzelmann-Schwarz et al., A distinct molecular profile associated with mucinous epithelial ovarian cancer, $\mathrm{Br} J$ Cancer 94(6) (2006), 904-913. 
[40] S. Hammarstrom, The carcinoembryonic antigen (CEA) family: structures, suggested functions and expression in normal and malignant tissues, Semin Cancer Biol 9(2) (1999), 67-81.

[41] C. Grotzinger et al., LI-cadherin: a marker of gastric metaplasia and neoplasia, Gut 49(1) (2001). 73-81.

[42] M.E. Huflejt and H. Leffler, Galectin-4 in normal tissues and cancer, Glycoconj J 20(4) (2004), 247-255.

[43] R.D. Blumenthal, H.J. Hansen and D.M. Goldenberg, Inhibition of adhesion, invasion, and metastasis by antibodies targeting CEACAM6 (NCA-90) and CEACAM5 (Carcinoembryonic Antigen) Cancer Res 65(19) (2005), 8809-8817.

[44] A.M. Chan et al., Isolation of a novel oncogene, NET1, from neuroepithelioma cells by expression cDNA cloning, Oncogene 12(6) (1996), 1259-1266.

[45] G. Sithanandam et al., Inactivation of ErbB3 by siRNA promotes apoptosis and attenuates growth and invasiveness of human lung adenocarcinoma cell line A549, Oncogene 24(11) (2005), 1847-1859.

[46] L.Y. Bourguignon et al., Hyaluronan promotes CD44v3-Vav2 interaction with Grb2-p185(HER2) and induces Rac1 and Ras signaling during ovarian tumor cell migration and growth, $J$ Biol Chem 276(52) (2001), 48679-48692.

[47] H.J. An et al., Comparative proteomics of ovarian epithelial tumors, J Proteome Res 5(5) (2006), 1082-1090.

[48] R.H. Young and R.E. Scully, Metastatic tumors in the ovary: a problem-oriented approach and review of the recent literature, Semin Diagn Pathol 8(4) (1991), 250-276.

[49] J. Prat, Ovarian carcinomas, including secondary tumors: diagnostically challenging areas, Mod Pathol 18(Suppl 2) (2005), S99-S111.

[50] H. Ji et al., Cytokeratins 7 and 20, Dpc4, and MUC5AC in the distinction of metastatic mucinous carcinomas in the ovary from primary ovarian mucinous tumors: Dpc4 assists in identifying metastatic pancreatic carcinomas, Int J Gynecol Pathol 21(4) (2002), 391-400.
[51] Y. Miyoshi et al., Somatic mutations of the APC gene in colorectal tumors: mutation cluster region in the APC gene, Hum Mol Genet 1(4) (1992), 229-233.

[52] Y.Y. Chou et al., Differentiation of ovarian mucinous carcinoma and metastatic colorectal adenocarcinoma by immunostaining with beta-catenin, Histopathology 43(2) (2003), 151156.

[53] E. Suh et al., A homeodomain protein related to caudal regulates intestine-specific gene transcription, Mol Cell Biol 14(11) (1994), 7340-7351.

[54] E. Suh and P.G. Traber, An intestine-specific homeobox gene regulates proliferation and differentiation, Mol Cell Biol 16(2) (1996), 619-625.

[55] G. Gaggero et al., [Expression of the cdx 2 gene in benign intestinal-type mucinous ovarian tumors], Pathologica 95(4) (2003), 185-191

[56] S. Logani et al., Use of novel immunohistochemical markers expressed in colonic adenocarcinoma to distinguish primary ovarian tumors from metastatic colorectal carcinoma, Mod Pathol 18(1) (2005), 19-25.

[57] G.M. Groisman, A. Meir and E. Sabo, The value of Cdx2 immunostaining in differentiating primary ovarian carcinomas from colonic carcinomas metastatic to the ovaries, Int $J G y$ necol Pathol 23(1) (2004), 52-57.

[58] R. Vang et al., Immunohistochemical expression of CDX2 in primary ovarian mucinous tumors and metastatic mucinous carcinomas involving the ovary: comparison with CK20 and correlation with coordinate expression of CK7, Mod Pathol 19(11) (2006), 1421-1428.

[59] S. Lee et al., Differential expression in normal-adenomacarcinoma sequence suggests complex molecular carcinogenesis in colon, Oncol Rep 16(4) (2006), 747-754

[60] J.C. Byrd and R.S. Bresalier, Mucins and mucin binding proteins in colorectal cancer, Cancer Metastasis Rev 23(1-2) (2004), 77-99. 


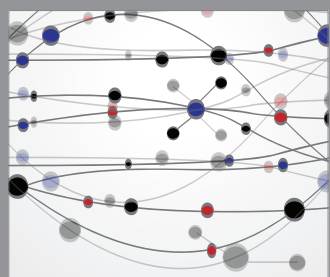

The Scientific World Journal
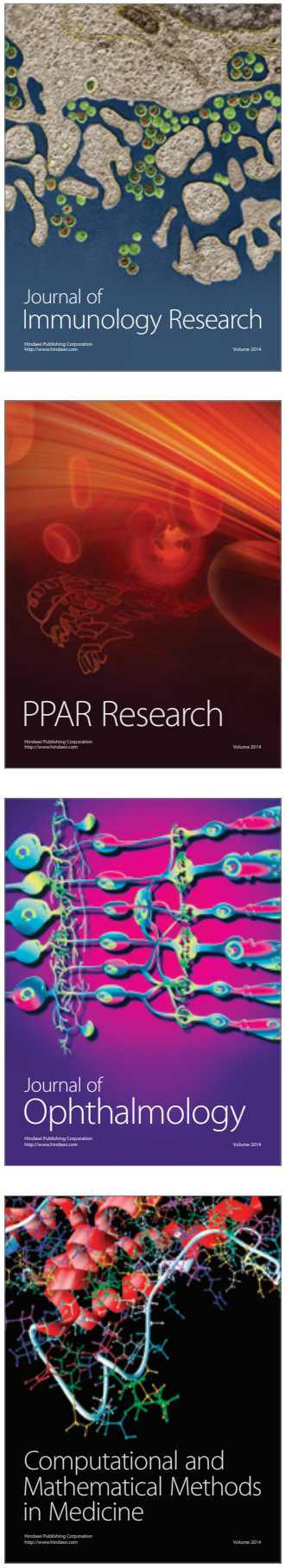

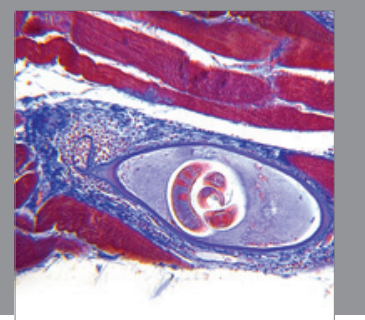

Gastroenterology

Research and Practice
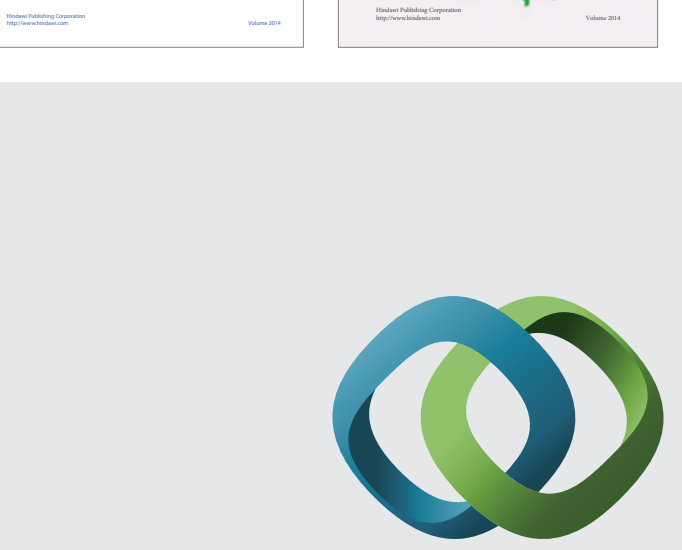

\section{Hindawi}

Submit your manuscripts at

http://www.hindawi.com
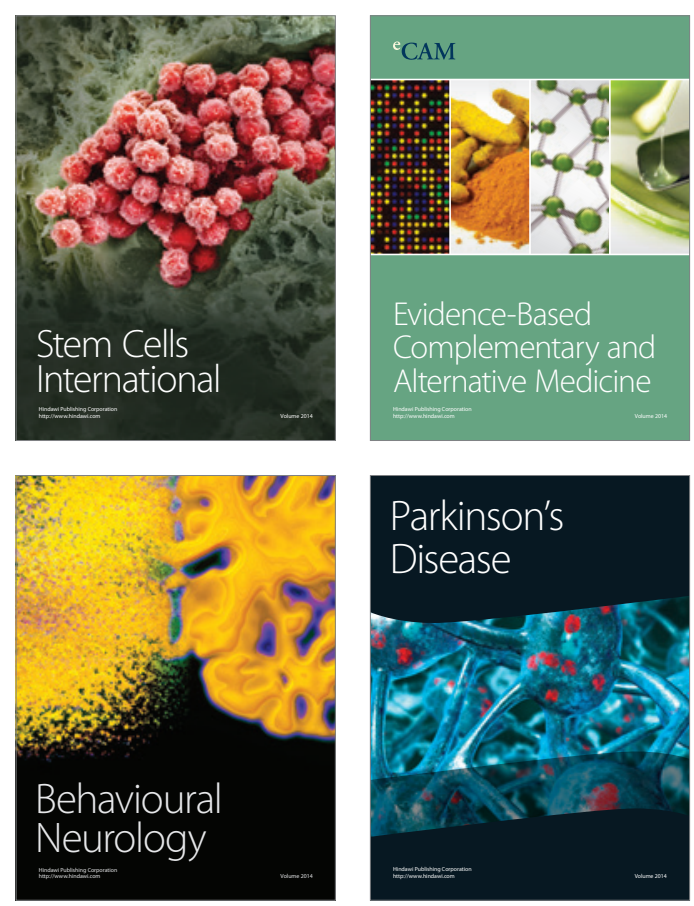

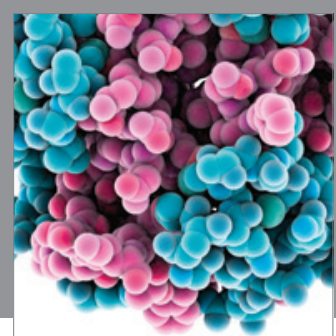

Journal of
Diabetes Research

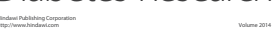

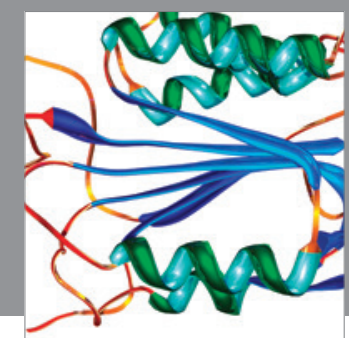

Disease Markers
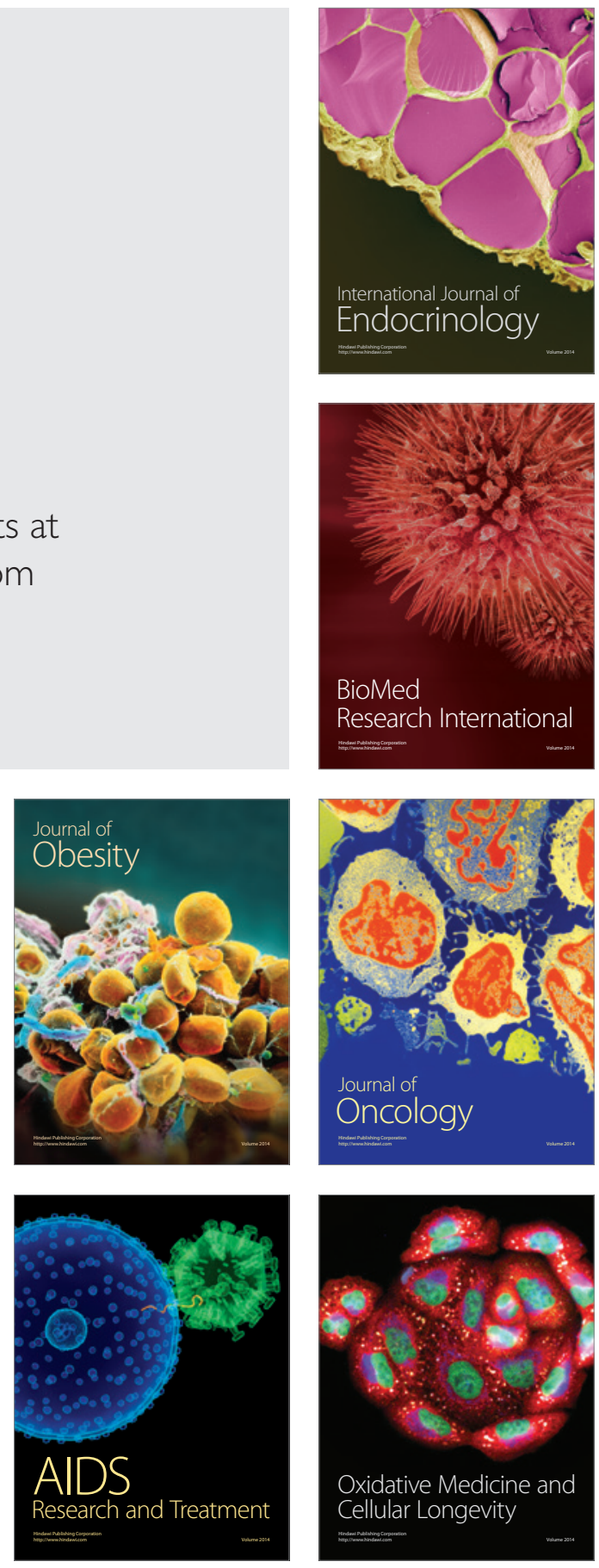